\title{
Fermeture de la communication et information d'expert
}

Laurence Monnoyer

\section{(2) OpenEdition}

1 Journals

Édition électronique

URL : http://journals.openedition.org/communicationorganisation/1815

DOI : 10.4000/communicationorganisation. 1815

ISSN : $1775-3546$

Éditeur

Presses universitaires de Bordeaux

Édition imprimée

Date de publication : 1 novembre 1995

ISSN : 1168-5549

Référence électronique

Laurence Monnoyer, « Fermeture de la communication et information d'expert », Communication et organisation [En ligne], 8| 1995, mis en ligne le 26 mars 2012, consulté le 19 avril 2019. URL : http:// journals.openedition.org/communicationorganisation/1815; DOI : 10.4000/ communicationorganisation. 1815

Ce document a été généré automatiquement le 19 avril 2019

(c) Presses universitaires de Bordeaux 


\title{
Fermeture de la communication et information d'expert
}

\author{
Laurence Monnoyer
}

1 Ce travail de recherche entamé dans le cadre de la thèse de doctorat a pour objectif la remise en cause de l'hypothèse habermassienne de la partition entre système et monde vécu à travers l'exemple de l'évolution des modes de légitimation du discours politique sur les essais nucléaires depuis le début de la Ve République en France. En particulier, il s'agit de mettre en évidence empiriquement que les processus de légitimation du discours politique ne s'épuisent pas dans les catégories analytiques définies par Habermas et qu'ils ne se trouvent pas d'explication satisfaisante dans la dichotomie entre système et monde vécu. Nous verrons successivement comment Habermas construit sa théorie de la légitimité autour de cette partition; puis on verra quelles sont les limites théoriques à son raisonnement dans le cadre particulier de la relation entre système politique et monde vécu; enfin, on posera quelques jalons expérimentaux qui devraient permettre d'appuyer nos critiques.

\section{Types de rationalité et partition habermassienne}

2 La théorie de la communication d'Habermas ${ }^{1}$ dans sa phase de maturité se présente en effet comme une théorie sociétale de la communication dont l'apport principal consiste à voir à l'œuvre dans la réalité sociale deux types de rationalités qui orientent les actions des acteurs sociaux. La première s'inspire des travaux de T. Parsons ${ }^{2}$ qui analyse la société comme un ensemble de systèmes dont les interactions transitent par des médias régulateurs que sont la monnaie, le pouvoir, l'engagement par rapport aux valeurs, etc. Ce type de rationalité est dit instrumental dans la mesure où les rapports intrasystémiques et inter-systémiques s'analysent comme des échanges stratégiques nondiscursifs orientés vers la réalisation des buts internes au système. Le développement de la rationalité instrumentale correspond pour Habermas à la complexification des sociétés industrielles qui, pour faire face tant aux attentes croissantes du monde vécu qu'aux 
pressions accrues qui résultent de l'internationalisation des marchés, s'organise en systèmes autonomes-Habermas en retient trois principaux : le système économique, le système administratif/politique et le système juridique, et dont la caractéristique principale est la recherche de l'efficacité maximale en dehors de toute intervention normative de la part des acteurs du système-La régulation des actions au sein des systèmes passe par les médias du pouvoir, de la monnaie et de la légalité, et dépasse la conscience des acteurs.

3 La seconde rationalité à l'œuvre est communicationnelle. Les normes d'action sont ici basées sur l'intercompréhension et l'échange discursif. Habermas s'inspire pour cette forme de rationalité des développements de la pragmatique du langage de Wittgenstein et de Pierce et le voit à l'œuvre au sein du "monde vécu », concept réactualisé de l'espace public qu'il avait analysé dans son célèbre ouvrage L'Espace public.

4 Les sociétés modernes se caractérisent donc par une partition entre deux sphères aux fonctionnements opposés, l'une systémique, dont les actions sont régies par la rationalité instrumentale et l'autre asystémique dont les actions sont régies par la rationalité communicationnelle. Les socio-pathologies de la vie moderne sont, selon Habermas, dues à la subordination croissante du monde vécu aux impératifs systémiques de la reproduction matérielle. Le concept de colonisation du monde vécu traduit cette tendance contemporaine à la complexification par l'extension des systèmes à la sphère publique jusque-là dominée par les prémisses communicationnels.

5 Ainsi il montre dans Raison et légitimités comment la sphère politique (les concepts de système et de monde vécu n'étaient qu'embryonnaires dans sa théorie d'alors) se déconnecte progressivement de ses bases légitimantes sous le double effet de l'interventionnisme croissant de l'État et d'une civilisation des loisirs qui n'engage pas à la participation politique. Ce qu'Habermas cherche ici à mettre en évidence est la matérialisation des rapports entre le citoyen et l'État : celui-ci doit assurer au premier des conditions de vie agréables, en échange de quoi le citoyen est prêt à payer davantage d'impôts mais surtout prêt à le laisser intervenir dans des domaines jusque-là réservés à l'initiative individuelle. Il en résulte une diminution des échanges normatifs entre les deux sphères : le citoyen se contente de demander biens et services, son silence faisant office de satisfaction alors que l'État, en multipliant ses domaines d'intervention, aurait besoin d'une ressource légitimante accrue. Habermas en déduit que les sociétés capitalistes avancées doivent faire face à une crise de légitimité qui ne saurait se résoudre sans une participation politique accrue, un soutien normatif fort de la part des populations sur lesquelles il exerce un pouvoir croissant. Or, souligne l'auteur, les populations concernées ne sont plus aptes à fournir ces ressources légitimantes dont l'État social a tant besoin. Pour plusieurs raisons: d'une part, la rationalisation instrumentale qui accompagne le développement des systèmes prend progressivement le pas, au sein même du monde vécu, sur la rationalité communicationnelle. En conséquence, l'espace public ne sait plus générer par l'intercompréhension les normes et les valeurs susceptibles de fournir les ressources légitimantes nécessaires au système. On y reconnaît la tendance wéberienne au désenchantement. D'autre part, la réserve de traditions dont ont vécu dans le capitalisme libéra l'État et le système du travail est épuisé : les éléments essentiels de l'idéologie bourgeoise deviennent incertains (mise en péril du privatisme dans la vie publique, dans la famille et dans la vie professionnelle) et les éléments résiduels de l'idéologie bourgeoise (la foi en la science, les systèmes de valeurs universalistes) constituent un cadre normatif dysfonctionnel. Le capitalisme 
avancé fait naître des besoins nouveaux qu'il ne peut satisfaire. C'est ce qu'Habermas appelle La crise de motivation.

\section{À la recherche d'une légitimité politique}

6 Habermas ne déduit certes pas de cette partition l'absence total d'échanges entre système et monde vécu. En particulier, le système politique reste fortement ancre dans le monde vécu : le média "pouvoir» assure la transmission à l'espace public des décisions du système politique. Par ailleurs, la sphère politique a besoin pour son propre maintien $\mathrm{du}$ soutien du monde vécu, en particulier au sein des régimes démocratiques. Ce processus de légitimation emprunte d'une part le média « pouvoir» et s'exprime à travers la vote pendant les élections, et d'autre part, il consiste en la transmission au système politique d'un ensemble de normes et de valeurs (les «structures normatives» d'Habermas) qui légitiment plus largement l'organisation politique de la société et les grands choix effectués par les responsables au pouvoir.

7 Selon l'auteur cependant, les moyens d'expression du public se trouvent aujourd'hui largement remis en cause et singulièrement diminués. D'une part, nous l'avons vu, la crise de motivation qui frappe les sociétés du Capitalisme avancé ne permet plus l'émergence de structures normatives susceptibles de fournir au système Politique les ressources légitimantes dont il aurait besoin. En ce sens, l'éthique de la discussion qu'il appelle de ces vœux ne trouverait plus les moyens de se réaliser au sein de l'espace public. Le système politique se retrouverait donc relativement fermé sur lui-même et contraint de trouver des palliatifs à cette légitimité qui lui ferait tant défaut. Les moyens de communication actuels Permettraient de développer une légitimation artificielle qui passerait par une fausse transparence de l'État et des techniques de mobilisation de l'opinion par le biais des sondages d'approbation de l'action gouvernementale. Une autre technique utilisée consiste, par ailleurs, à appeler au secours de la sphère politique des experts et des scientifiques qui apporteraient leur crédit aux actions engagées par les pouvoirs publics. Cette forme de légitimation de l'autorité politique a le grand avantage de couper court - relativement - aux discussions au sein de l'espace public dans la mesure où les citoyens ne disposent que difficilement de l'information suffisante Pour venir contredire les rapports des experts.

8 Face à ce type de comportement, les citoyens ne disposent que de faibles moyens d'intervention. D'une Part, l'action des médias constitue une sorte d'écran entre la représentation nationale et les citoyens et remplace Progressivement l'espace public, espace de discussion et de structuration des valeurs et des normes. D'autre part, ces mêmes médias de masse, qui auraient pu former une sorte d'espace de discussion élargi et largement ouvert à l'intervention populaire, ont pris une dimension consumériste qui relève plus de la stratégie de marketing que de la tribune d'expression populaire. Dans cette civilisation des loisirs qui caractérise les sociétés du capitalisme avancé, les lieux de discussion ont disparu, favorisant ainsi une fermeture du système politique sur lui-même.

Le discours d'Habermas peut apparaître bien pessimiste. Il reconnaît cependant lui-même que le monde vécu n'a pas totalement perdu son pouvoir d'expression-Le sociologue de Francfort reconnaît que ça et là des associations diverses maintiennent des espaces d'échanges discursifs au sein desquels la rationalite communicationnelle basée sur l'intercompréhension fait face au développement du système. Cette résistance n'est toutefois pas organisée de façon cohérente et se trouve dans une situation de faiblesse 
face à la pression croissante des impératifs systémiques. Sur le point de savoir comment ces ressources légitimantes peuvent influencer le système politique, Habermas ne dit mot. Et c'est sans doute là la plus grande faiblesse de la théorie de l'agir communicationnel.

En réduisant au média "pouvoir » les interactions entre système et monde vécu, Habermas ne peut en effet expliquer ni comment les normes et les valeurs pénètrent dans le système politique, ni pourquoi une diminution des ressources légitimantes affecterait le système. La participation des citoyens se résumant dans sa théorie à leur capacité de voter (média "pouvoir»), on ne voit pas comment les structures normatives emprunteraient cette voie pour venir légitimer l'action politique. Autrement dit, l'activité d'intercompréhension, si elle structure bien les relations dans le monde vécu et génère bien des valeurs fondamentales d'une société, peut-elle trouver un média de transition vers la sphère politique ? Comme le souligne bien $\mathrm{H}$. Baxter, à refuser d'utiliser le média parsonnien de Value commitment qui organise les relations entre système culturel et système politique, Habermas se prive du moyen d'analyser en détail les processus de légitimation du politique. Sur le fond, il conçoit bien qu'une organisation politique est étroitement liée au système culturel dans lequel elle se développe, mais sur la forme, rien ne permet d'expliquer concrètement comment ce processus de légitimation s'organise. De ce fait, toute la construction bipartite devient problématique. Peut-on dire que le système politique, basé sur l'action instrumentale, est finalement sensible à une autre forme de rationalité, communicationnelle cette fois? Mais, dans ce cas, y-a-t-il encore un sens à parler de séparation stricte entre système et monde vécu?

La crise de légitimité à laquelle Habermas a consacré tant de développements peut dès lors apparaître comme un effet pervers de sa construction théorique : n'ayant pas prévu les concepts théoriques pour penser la légitimité, il en conclut qu'elle a disparu!

L'objectif de la thèse reste réaliste et il ne s'agit pas d'apporter une réponse aux multiples questions qui se posent ici. Nous voudrions seulement montrer sur un exemple - pratique auquel malheureusement Habermas et ses critiques ont très peu recours, que les processus de légitimation ne répondent pas au modèle habermassien.

\section{L'expert, le politique et les essais nucléaires}

La partie expérimentale n'étant pas achevée, on se contentera ici de donner une estimation des résultats obtenus.

L'idée sous-jacente à la méthodologie utilisée est la suivante - ; il s'agit de montrer :

1 - Que l'expertise scientifique, indice de relative fermeture du discours politique, et donc facteur bloquant du processus de légitimation, ne constitue pas un barrage définitif au processus.

162 - Qu'il existe bel et bien au sein du monde vécu, des associations et des leaders d'opinion qui continuent à vouloir influer sur le système politique.

173 - Que des actions sont engagées dans l'espace public dans ce sens, et parfois avec une certaine efficacité, ou du moins qu'elles obligent le gouvernement à revoir sinon le contenu des décisions, en tous cas les formes d'élaboration de la décision.

184 - Que ces actions constituent une forme d'expression légitimante dont le système politique doit historiquement tenir compte. 
19 Sur le plan théorique, si l'on parvient à apporter des réponses sur ces quatre points, on aura montré que la partition habermassienne entre système et monde vécu ne permet pas de rendre compte du processus de légitimation dans les sociétés modernes.

Pour ce faire, nous avons choisi de nous intéresser au discours politique concernant les essais nucléaires. L'avantage de ce choix est double: d'une part, il permet une mise en perspective historique, la politique nucléaire remontant aux débuts de la Ve République. Par ailleurs, il s'agit d'un sujet relativement controversé qui autorise une analyse en profondeur des débats qui se déroulent au sein de l'espace public.

21 Le corpus se compose d'une part de l'ensemble des articles parus dans Le Monde et dans Le Figaro sur le sujet depuis 1958, et d'autre part de l'ensemble des discours d'investiture des Premiers Ministres devant l'Assemblée Nationale depuis que cette tradition parlementaire existe, c'est-à-dire 1974. Des entretiens avec quelques spécialistes de la question (membres d'associations, scientifiques engagés) sont également prévus ou partiellement réalisés. lieu. Il semble que cette Pratique se renforce dans les quinze dernières années, à mesure que le besoin de confirmation de la non-nocivité écologique des essais se fait sentir. Toutefois, les associations se dotent également d'autorités scientifiques susceptibles de répondre par des contre-expertises aux allégations des experts du gouvernement. Le débat en ce sens se technicise mais ne disparaît pas pour autant. Au contraire, les discussions entre experts occupent une grande place des querelles entre l'État et les associations concernées.

pression populaire contraint le gouvernement à adopter une stratégie de communication plus ouverte qu'auparavant et il se voit désormais refuser l'excuse de secret défense » jusque-là invoquée. La récente décision de suspension des essais nucléaires après seulement six des huit essais jusque-là annoncés semble aller dans le sens d'une plus grande prise en compte qu'auparavant des revendications populaires.

$$
\text { haut. Il faudra bien sûr attendre les résultats définitifs pour s'avancer davantage. }
$$
à savoir quels sont les concepts qui permettraient une analyse plus rigoureuse des processus de légitimation, ils offrent de belles perspectives de recherches pour les années qui viennent... 


\section{NOTES}

1. Habermas J.., Théorie de l'agir communicationnel, Paris, Fayard, 1987, 2 tomes.

2. Parsons T., The structure of social action, New York, The Free Press, $2^{\mathrm{e}}$ édition, 1949.

3. Habennas J., Raison et légitimité, Problèmes de légitimité dans le capitalisme avancé, Paris, Payot, 1973.

\section{RÉSUMÉS}

L'article suivant s'appuie sur un travail de thèse en cours. Il tente de montrer que les processus de légitimation du discours politique ne s'épuisent pas dans les catégories analytiques définies par Habermas : système et monde vécu. Une expérimentation sur un discours théorique qui, indirectement, renvoie à la question de l'information, assénée ou justifiée, et de la communication au sens le plus noble du terme : la discussion. Avec une évidence sous-jacente : il n'y a de contrepoids à la fermeture du système politique que l'information citoyenne.

The following article is based on work for a thesis that is currently being written. It attempts to show that the processes whereby political discourse are legitimised are not exhaustively treated in the analytical categories defined by Habermas: system and the world experienced. This experiment around a theoretical discourse indirectly comes back to the question of information imposed without explanation or justification and communication as discussion, which is the noblest sense of the word. There is an underlying truth: there is no other counterweight to the closing up of the political system than making information available to the citizen.

\section{AUTEUR}

\section{LAURENCE MONNOYER}

Laurence Monnoyer est ATER. Elle est membre de l'équipe Culture. Technologies et Pratiques Sociales du Laboratoire d'Etudes et de Recherches Appliquées en Sciences Sociales (LERASS) à l'IUT Paul Sabatier de Toulouse. Son travail de thèse en cours porte sur les nouveaux modes de légitimation du discours politique, à travers une analyse de ce type de discours à propos des essais nucléaires depuis les débuts de la Ve République. L'objectif théorique de la recherche vise à s'interroger sur la pertinence heuristique des concepts habermassiens de Système et de Monde vécu dans leur application au politique. 